\title{
Alternative Form of Ordinary Differential Equation of Electroencephalography Signals During an Epileptic Seizure
}

\author{
Ameen Omar Ali Barja ${ }^{a, b, *}$ \\ aDepartment of Mathematics, College of Education, Seiyun University, Hadhramout,

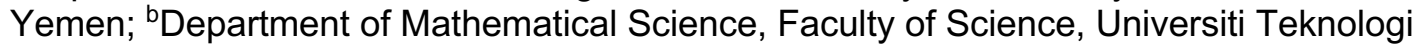 \\ Malaysia, 81310 Skudai, Johor, Malaysia
}

\begin{abstract}
One of the most important fields in clinical neurophysiology is an electroencephalogram (EEG). It is a test used to detect problems related to the brain electrical activity, and it can track and records patterns of brain waves. EEG continues to play an essential role in diagnosis and management of patients with epileptic seizure disorders. Nevertheless, the outcome of EEG as a tool for evaluating epileptic seizure is often interpreted as a noise rather than an ordered pattern. The mathematical modelling of EEG signals provides valuable data to neurologists, and is heavily utilized in the diagnosis and treatment of epilepsy. EEG signals during the seizure can be modeled as ordinary differential equation (ODE). In this study we will present an alternative form of ODE of EEG signals through the seizure.
\end{abstract}

Keywords: EEG signals, ODE, initial time

*For correspondence: ameenbarja@seiyunu. edu.ye

Received: 26 June 2020

Accepted: 31 Jan 2021

(C) Copyright Barja. This article is distributed under the terms of the Creative Commons Attribution License, which permits unrestricted use and redistribution provided that the original author and source are credited.

\section{Introduction}

Epilepsy is a central nervous system (neurological) disorder in which brain activity becomes abnormal, causing seizures or periods of unusual behavior, sensations, and sometimes loss of awareness [1]. Epileptic seizure symptoms may vary widely, some patient having epilepsy merely stare blankly for a few seconds through a seizure, while others repeatedly twitch their arms or legs. Having a single seizure doesn't mean you have epilepsy. Leastways two unprovoked seizures are mostly necessary for an epilepsy diagnosis [2].

Treatment of the seizure can be by drugs or sometimes operation can control seizures for the majority of patients with epilepsy. Some patients need lifelong treatment to control seizures, but others, the seizures eventually go away [3]. EEG is an electrophysiological monitoring technique to record the brain spontaneous electrical activity over a period of time [see figure 1]. It is characteristically non-invasive, with the electrodes placed along the scalp, although invasive electrodes are sometimes used, as in electrocorticography [4].

The mathematical analysis of EEG signals during the seizure still relies, mostly, on its visual inspection. Due to the fact that visual inspection is actually very subjective and hardly allows any statistical analysis or standardization, several techniques were projected in order to quantify the data of the EEG. One of these techniques, Fourier Transform emerged as a very powerful tool capable of characterizing the frequency components of EEG signals, even reaching diagnostic importance. Nevertheless, Fourier 
Transform has some disadvantages that limit its applicability and therefore, other techniques for extracting hidden information from the EEG signals are required [5].

Feature space $\boldsymbol{F}$

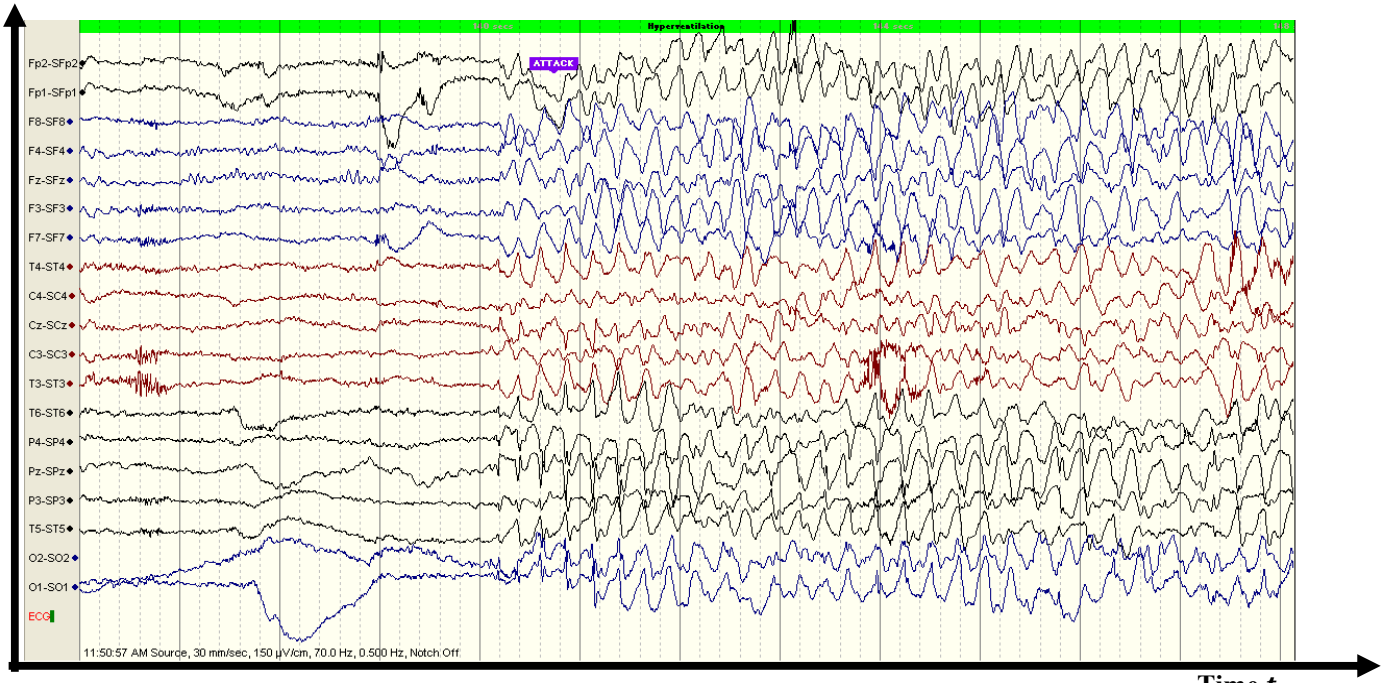

Fig 1: EEG signals of an epileptic seizure

Time $t$

To the moment, several techniques have been employed in the field of mathematical modelling of epileptic seizures, each with varying outcomes and goals. In 1986, Destexhe and Babloyantz have used the series of time for EEG records through a seizure to display the presence of deterministic dynamics of a complex nature for epileptic seizures [6]. By matching and classifying the chaotic attractors through ictic and between interictal seizures, they contended that an epileptic seizure is a low-dimensional state, which has been further simplified via Stam as a "complexity loss" [7]. Few years later this concept has been strengthened via lasemidis et al. by calculating the reduction of the largest Lyapunov exponent through a seizure [8], the outcome which has been further agreed in 1990 by Frank's EEG analysis of absence seizures, in which he actually indicated the existence of an underlying chaotic attractor [9].In the last decade, more mathematics modeling has been developed to extract hidden information from EEG data, for instance, in 2000, Ahmad et al. formulated a fuzzy-based topological model to classify the foci of an epileptic seizure [10]. The model, called Fuzzy Topographic Topological Mapping (commonly referred to by its abbreviation FTTM), involves containing a topology on the magnetic field of magnetoencephalographic recordings and utilizes fuzzy methods to estimate the location of the epileptic foci [11]. The epileptic foci here refer to points in the brain from which the seizures are assumed to originate. This technique has been further refined eight years later to contain EEG input [12].

Finally, another technique has been developed to extract more hidden information from EEG patient's data [13]; dynamical system modeling is a key tool to describe a seizure as a dynamic physical process or a continuous system which is represented by its motion. Furthermore, the representation can be modeled as an ordinary differential equation (ODE). By utilizing the previous finding, this study provides an alternative form of ODE of EEG signals during an epileptic seizure.

\section{Methods}

In this section, some existing definitions and /or outcomes that will be used in this paper are presented.

\section{Definition 1 [14]}

Let $\left(X, d_{X}\right)$ and $\left(\mathrm{T}, d_{Y}\right)$ be metric spaces, where $(\mathrm{T}, \prec)$ is a linearly ordered set with the minimal element $t_{0}$ in $\mathrm{T}$ and a linear order $\prec$. Let $X^{\mathrm{T}}$ be the set of all functions $f: \mathrm{T} \rightarrow X$. A dynamical system is a collection $\psi(t, x, y)$ of transformations of the space $X \times \mathrm{T} ;(x, y) \in X \times \mathrm{T}$, into $X^{\mathrm{T}}, \psi(., x, y) \in X^{\mathrm{T}}$ satisfying the conditions:

(I) $\psi\left(t_{0}, x, t_{0}\right)=x$

(II) $\psi\left(t, x, t_{0}\right)$ is defined for all $t$ 
(III) $\psi\left(t, x, t_{0}\right)$ is unique , i.e. $\psi\left[t, \psi\left(t, x, t_{1}\right), t_{1}\right]=\psi\left(t, x, t_{0}\right)$ for all $t_{0}, t_{1}$

(IV) $\psi(t, x, y)$ is continuous in all arguments

The point $(x, y) \in X \times \mathrm{T}$ is called an initial condition. The function $\psi(., x, y)$ for a fixed $(x, y)$ is called a motion. The set of points $X=\left\{\psi\left(t, x, t_{0}\right) ; t \in \mathrm{T}\right\}$ is called a trajectory of this motion.

A continuous dynamical system is one of the types of the dynamical system, which is a system that has evolved over time and been denoted by the variable $t$, where $t$ is a real number, and it can be expressed as an ODE as follows

$$
\frac{d x}{d t}=\varphi(x) ; t \in \mathbb{R}
$$

where $x \in X$; represents the system state, $X$ being phase space [15].

\section{Definition 2 [16]}

Let $\psi$ is a unique trajectory at $x_{0}$. The flow of $\frac{d x}{d t}=\varphi(x) ; t \in \mathbb{R}$ is the map $\Phi: \Omega \rightarrow \mathrm{T}, \quad(t, x) \mapsto$ $\psi(t, x)$, where $\psi(t, x)$ is the maximal trajectory at $x$ defined on a maximal interval $I_{x}=\left(\mathrm{T}_{-}(x), \mathrm{T}_{+}(x)\right)$, and $\Omega$ is the set $\Omega=U_{x \in \mathrm{T}}\left(I_{x} \times\{x\}\right) \subseteq \mathbb{R} \times \mathrm{T}$, where $\mathrm{T}$ is an open subset of $\mathbb{R}^{n}$.

\section{Theorem 3 [15]}

Suppose $\psi \in X\left(U, \mathbb{R}^{n}\right)$, where $U$ is open subset of $\mathbb{R}^{n+1}$ is Lipschitz continuous. Around each point $\left(x_{0}, t_{0}\right) \in U$ we can find an open set $I \times V \subseteq U$ such that $\varphi(x, t) \in X\left(I \times V, \mathbb{R}^{n}\right)$, where $I$ is a compact interval and $V$ is a compact set.

\section{Theorem 4 [17]}

The signals of EEG during an epileptic seizure can be represented by the following ODE

$$
\left.\frac{d x}{d t}\right|_{\left(t, x_{0}, t_{0}\right)}=\psi\left(x\left(t, x_{0}, t_{0}\right), t\right) ; t \in \mathbb{R}
$$

where, if the equation 2 is a continuous time dynamical system during the seizure, then the function $\psi$ it should has a unique function that satisfy equation 2 starting at initial state $x_{0}$ and initial time $t_{0}$, where $x\left(t, x_{0}, t_{0}\right)$ is a dynamical system (i.e. $\phi(t) ; \forall t \in \mathbb{R}$ is a corresponding motion), and $\left(x_{0}, t_{0}\right)$ is a stationary point during the seizure.

\section{Alternative from ODE of EEG signals during the seizure}

Our main goal in this section is to show that equation 2 it can be transfer to alternative form based on vector fields. First, we have the following lemma:

\section{Lemma 1}

Suppose $\psi \in X^{\mathrm{T}}$. For all $x \in \mathrm{T}$ there exists an interval $I_{x} \subseteq \mathbb{R}$ containing 0 and a corresponding unique maximal trajectory $\Phi(., x) \in X^{\mathrm{T}}\left(I_{x}, \mathrm{~T}\right)$ at $x$. Moreover, the set $\Omega$ is open during the seizure and $\Phi \in$ $X^{\mathrm{T}}(\Omega, \mathrm{T})$ is flow during the seizure on $\mathrm{T}$, that is, $\Phi(0, x)=x, \Phi(\alpha+t, x)=\Phi(t, \Phi(\alpha, x)), x \in \mathrm{T}, \alpha, \alpha+$ $t \in I_{x} ; t$ is a real time of EEG signals.

\section{Proof}

Assume that $\left(x_{0}, t_{0}\right) \in \Omega$ is a stationary point (implying $\left.t_{0} \in I_{x_{0}}\right)$ and set $\omega=\Phi_{x_{0}}\left(\left[0, t_{0}\right]\right)$. By theorem 3 there exists an open neighborhood $(-\epsilon(x), \epsilon(x)) \times U(x)$ of $(0, x)$ around each point $x \in \omega$ such that $\Phi$ is defined and $X^{\mathrm{T}}$ on this neighborhood. Since $\omega$ is compact, finitely many of this neighborhood cover $\{0\} \times \omega$. Thus there exists $\epsilon>0$ and an open neighborhood $U$ of $\omega$ such that $\Phi$ is defined on $(-\epsilon, \epsilon) \times U$. Next, let $z \in \mathbb{Z}^{+}$so large that $\frac{t_{0}}{z}<\epsilon$ and pick $\mathrm{K}^{j}(x)=\mathrm{K}\left(\mathrm{K}^{j-1}(x)\right)$, where $\mathrm{K}(x)=\Phi_{\frac{t_{0}}{z}}(x)$ is $X^{z}$ for $x \in U$ by construction. Since $\mathrm{K}^{j}\left(x_{0}\right) \in \omega \subset U$ for $1 \leq j \leq z$, there exists an open neighborhood $U_{0} \subseteq U$ of $x_{0}$ such that $\mathrm{K}^{z}$ is defined on $U_{0}$. Furthermore, $\Phi(t, x)=\Phi\left(t-t_{0}, \Phi\left(t_{0}, x\right)\right)=\Phi\left(t-t_{0}, \mathrm{~K}^{z}(x)\right)$ is defined and smooth for all $(t, x) \in\left(t_{0}+\epsilon, t_{0}-\epsilon\right) \times U_{0}$ where, $t_{0}$ is initial time and $x_{0}$ is initial state of equation 2 . 


\section{Theorem 2}

Suppose $\psi\left(x_{0}\right) \neq 0$. Then there exists a local coordinate transform $y=\varphi(x)$ such that the ODE 2 of EEG signals during the seizure can be transformed to the form $y^{\prime}=(1,0, \ldots, 0)$ at any time.

\section{Proof}

Consider all points starting on the feature space $x_{1}=0$. Then the point $\Phi\left(t,\left(0, x_{2}, \ldots, x_{n}\right)\right)$ should be mapped to the point $\left(0, x_{2}, \ldots, x_{n}\right)+t(1,0, \ldots, 0)=\left(t, x_{2}, \ldots x_{n}\right)$. Hence the inverse of the map we are looking for should be given by

$$
\zeta(x)=\Phi\left(x_{1},\left(0, x_{2}, \ldots, x_{n}\right)\right)
$$

which is well defined in a neighborhood of 0 . The Jacobi determinant at 0 is given by

$$
\left.\operatorname{det}\left(\frac{\partial \zeta_{i}}{\partial x_{j}}\right)\right|_{x=0}=\left.\operatorname{det}\left(\frac{\partial \Phi}{\partial t}, \frac{\partial \Phi}{\partial x_{2}}, \ldots, \frac{\partial \Phi}{\partial x_{n}}\right)\right|_{t=o, x=0}=\operatorname{det} \mathbb{1}_{n}=1
$$

Since $\partial \Phi /\left.\partial x\right|_{t=0, x=0}=\mathbb{1}_{n}$ and $\partial \Phi /\left.\partial t\right|_{t=0, x=0}=\psi(0)=(1,0, \ldots, 0)$ by assumption. So by the inverse function theorem we can assume that $\zeta$ is a local diffeomorphism and we can consider new coordinates $y=\zeta^{-1}(x)$. Since $\partial \zeta_{j} / \partial x_{1}=\psi_{j}(\zeta(x))$ our system reads in the new coordinates

$$
y_{j}^{\prime}=\left(\frac{\partial \zeta_{j}}{\partial x_{i}}\right)_{\zeta^{-1}(x)}^{-1} \psi_{i}(x)=\delta_{1, j}
$$

Which is the required form.

\section{Discussion}

Epileptic seizure is an extremely complex dynamic process due to its nature in exhibiting high variability i.e., high number of state variables. However, dynamical system model of EEG signals can be a good platform to represent the seizure dynamically. As a matter of fact, the previous study was initial point to represent EEG signals during the seizure as ODE. The vectors field in mathematics is a string and direct way to view a novel representation of the ODE of EEG signals during the seizure and make it much straight and stable.

\section{Conclusion}

This paper presented an alternative form of ODE of EEG signals during an epileptic seizure using vectors field. In general, the current results may assist us to thoroughly examine features of EEG signals during an epileptic seizure.

\section{References}

[1] Fisher, R.S., et al., ILAE official report: a practical clinical definition of epilepsy. Epilepsia, 2014. 55(4): p. 475482.

[2] Duncan, J.S. and H.J. Cross, Epilepsy and the overlap with autonomic disorders. Autonomic Failure: A Textbook of Clinical Disorders of the Autonomic Nervous System, 2013: p. 435.

[3] Nadkarni, S., J. LaJoie, and O. Devinsky, Current treatments of epilepsy. Neurology, 2005. 64(12 suppl 3): p. S2-S11.

[4] Niedermeyer, E., D.L. Schomer, and F.H.L. da Silva, Niedermeyer's Electroencephalography: Basic Principles, Clinical Applications, and Related Fields. 2011: Wolters Kluwer Health/Lippincott Williams \& Wilkins.

[5] Quiroga, R.Q., Quantitative analysis of EEG signals: time-frequency methods and chaos theory. Institute of Physiology-Medical University Lubeck and Institute of Signal Processing-Medical University Lubeck, 1998.

[6] Babloyantz, A. and A. Destexhe, Low-Dimensional Chaos in an Instance of Epilepsy. Proceedings of the National Academy of Sciences, 1986. 83(10): p. 3513-3517.

[7] Stam, C.J., Nonlinear Dynamical Analysis of EEG and MEG: Review of an Emerging Field. Clinical Neurophysiology, 2005. 116(10): p. 2266-2301. 
[8] lasemidis, L.D., et al., Phase space Topography and the Lyapunov Exponent of Electrocorticograms in Partial Seizures. Brain Topography, 1990. 2(3): p. 187-201.

[9] Frank, G., et al., Chaotic Time Series Analyses of Epileptic Seizures. Physica D: Nonlinear Phenomena, 1990. 46(3): p. 427-438.

[10] Ahmad, T., et al., Development of Detection Model for Neuromagnetic Fields. Proceedings of the BIOMED, 2000: p. 119-121.

[11] Ahmad, T., et al., Fuzzy Topographic Topological Mapping for Localisation Simulated Multiple Current Sources of MEG. Journal of Interdisciplinary Mathematics, 2008. 11: p. 381-393.

[12] Ahmad, T., et al. Selection of A subset of EEG Channels of Epileptic Patient During Seizure Using PCA. in Proceedings of the 7th WSEAS International Conference on Signal Processing, Robotics and Automation. 2008. World Scientific and Engineering Academy and Society (WSEAS).

[13] Ahmad, T., et al. Dynamical System of an Epileptic Seizure. in International Conference on New Techniques in Pharmaceutical and Biomedical Research. 2005. Kuala Lumpur, Malaysia: IEEE.

[14] Kasanovich, B.R., Signal and System Analysis in Fuzzy Infonnation Space. 1995, University of Pittsburgh: United States of America.

[15] Teschl, G., Ordinary Differential Equations and Dynamical Systems. Vol. 140. 2012: American Mathematical Soc.

[16] Bhatia, N.P. and G.P. Szegö, Dynamical Systems: Stability Theory and Applications. 2006: Springer Berlin Heidelberg.

[17] Barja, A.O.A., Epileptic Seizure as a System of Ordinary Differential Equation, in Mathematics. 2012, Universiti Teknologi Malaysia: UTM. 